\title{
BMJ Open The Alberta Pregnancy Outcomes and Nutrition (APrON) longitudinal study: cohort profile and key findings from the first three years
}

\author{
Nicole Letourneau (D) , ${ }^{1,2}$ Fariba Aghajafari, ${ }^{3}$ Rhonda C Bell (D) , ${ }^{4}$ Andrea J Deane, ${ }^{5}$ \\ Deborah Dewey, ${ }^{5}$ Catherine Field, ${ }^{4}$ Gerald Giesbrecht, ${ }^{6}$ Bonnie Kaplan (D) , \\ Brenda Leung, ${ }^{7}$ Henry Ntanda, ${ }^{5}$ APrON Study Team
}

To cite: Letourneau N, Aghajafari F, Bell RC, et al. The Alberta Pregnancy Outcomes and Nutrition (APrON) longitudinal study: cohort profile and key findings from the first three years. BMJ Open 2022;12:e047503. doi:10.1136/ bmjopen-2020-047503

- Prepublication history for this paper is available online. To view these files, please visit the journal online (http://dx.doi org/10.1136/bmjopen-2020047503).

Received 11 December 2020 Accepted 09 November 2021

Check for updates

(C) Author(s) (or their employer(s)) 2021. Re-use permitted under CC BY-NC. No commercial re-use. See rights and permissions. Published by BMJ.

For numbered affiliations see end of article.

Correspondence to

Dr Nicole Letourneau;

nicole.letourneau@ucalgary.ca

\section{ABSTRACT}

Purpose The objectives of the ongoing Canadian Iongitudinal cohort called the Alberta Pregnancy Outcomes and Nutrition (APrON) study are to: (1) determine the relationship between maternal nutrient intake and status before, during, after pregnancy, and (a) maternal mental health, (b) pregnancy and birth outcomes, and (c) infant/ child neurodevelopment and behavior; (2) identify maternal mental health and nutrient predictors of child behaviour; and (3) establish a DNA biobank to explore genomic predictors of children's neurodevelopment and behavior. The purpose of this paper is to describe the participants, measures, and key findings on maternal and paternal mental health, maternal nutrition, and child outcomes to when children are 3 years of age.

Participants Participants included mothers and their children $(n=2189)$ and mothers' partners (usually fathers; $\mathrm{n}=1325$ ) from whom data were collected during the period from pregnancy to when children were 3 years of age, in Alberta, Canada. More than $88 \%$ of families have been retained to take part in completed data collection at 8 years of age.

Findings to date Data comprise: questionnaires completed by pregnant women/mothers and their partners on mothers', fathers' and children's health; dietary interviews; clinical assessments; linkage to hospital obstetrical records; and biological samples such as DNA. Key findings on mental health, nutrition and child outcomes are presented. APrON women who consumed more selenium and omega- 3 were less likely to develop symptoms of perinatal depression. Higher prenatal consumption of choline rich foods such as eggs and milk were recommended as was vitamin $\mathrm{D}$ supplementation for both mothers and children to meet guidelines. Couples in which both mothers and fathers were affected by perinatal depression reported lower incomes and higher maternal prenatal depressive symptoms and lower support from fathers postnatally and their children presented with the most behavioural problems. Maternal experiences of early adversity predicted increased likelihood of perinatal depression and anxiety and children's behavioural problems.

Future plans The APrON cohort offers a unique opportunity to advance understanding of the developmental origins of health and disease. There is a planned follow-up to collect data at 12 years of age.
Strengths and limitations of this study

- APrON is a large, ongoing longitudinal study designed to understand the early origins of health and disease.

- APrON has collected data to 8 years of age and has planned follow-up at 12 years of age.

- APrON has retained more than $88 \%$ of participants at the 8-year data collection.

- A limitation is that the cohort is largely low-risk, limiting generalisability of study findings to higher-risk populations.

\section{WHY WAS THE APRON COHORT SET UP?}

The Alberta Pregnancy Outcomes and Nutrition (APrON) study is a longitudinal cohort of pregnant women, their partners, and their children, in Alberta, Canada. Women in our study are biological mothers, and partners typically self-identified as fathers of their children whether biological or not. The APrON study was designed to investigate the effect of nutrient intake and status during pregnancy on maternal mental health and children's neurodevelopment and behaviour by meeting three objectives. ${ }^{12}$ First, the APrON team sought to determine the relationship between maternal nutrient intake and status before, during, and after pregnancy, and (a) maternal mental health, (b) pregnancy and birth outcomes, and (c) infant/child neurodevelopment and behaviour. Second, the team sought to identify maternal mental health and nutrient predictors of child behaviour over time. Third, the team sought to establish a DNA biobank to explore genomic predictors of children's neurodevelopment and behaviour. An original grant of $\$ 5$ million provided the foundation for the APrON study, which has subsequently 
expanded its focus and contributed to over 50 additional projects on the psychobiology of stress, parentchild relationships and attachment, neurotoxicant exposure, genetics, epigenetics, inflammation and immune activity, probiotics, the microbiome, and children's brain development. To date, the APrON study has received over $\$ 15$ million in additional funding. This funding enables follow-up of APrON families until children reach 12 years of age to understand the impact of medical, biological and environmental factors on children's development, behaviour and mental health. Data collection for the 5-year and 8-year follow-up questionnaires is completed, with the data currently being cleaned for analysis and the 12-year follow-up to begin in 2022. Papers have been published detailing how women and their partners were recruited ${ }^{23}$ and describing the APrON cohort at enrollment during pregnancy ${ }^{45}$ and up to 12 months postpartum ${ }^{1}$; however, a summary of key findings has not been reported. Thus, drawn mostly from data collected during the period from pregnancy to when children are 3 years of age, the objectives of this paper are to describe the: (1) participants, (2) measures, and (3) key findings on maternal and paternal mental health, maternal nutrition and child outcomes.

\section{WHO IS IN THE APRON COHORT?}

Women were recruited into the cohort if they were 16 years of age or older, able to speak and read English, $<27$ weeks gestation and willing to come for on-site clinic visits at the University of Calgary, in Calgary or University of Alberta, in Edmonton, Alberta. Partners were eligible if they were cohabitating with the participating woman. Participants were excluded if they planned to move out of the region during the clinic visit timeframe of pregnancy to 3 months postpartum. A total of 2189 pregnant women (aged 16-44) and 1325 men (aged 18-52), residing around Calgary or Edmonton, were enrolled in the study between May 2009 and June 2012. To assess the representativeness of the APrON cohort (see table 1), sociodemographic characteristics of participants at the time of recruitment were compared with characteristics of women with children in Calgary, Alberta and Canada. ${ }^{5-7}$ A greater proportion of women in the cohort (28.0\%) were over 34 years old compared with women in Calgary $(20.0 \%)$, Alberta $(16.0 \%)$ and Canada $(18.0 \%)$. More APrON women were married $(95 \%)$ compared with women in Calgary $(73.0 \%)$, Alberta $(70.0 \%)$ and Canada $(60.0 \%)$. A greater proportion of women had household incomes of $\$ 60000$ or more $(77.0 \%)$ compared with Calgary $(65.0 \%)$, Alberta $(61.0 \%)$ and Canada (58.0\%). More APrON women completed post-secondary education $(87.0 \%)$ compared with women in Calgary $(69.0 \%)$, Alberta $(70.0 \%)$ and Canada $(72.0 \%)$. The proportion of APrON women who were born outside of Canada (22.0\%) was similar to the proportions in Calgary (25.0\%), Alberta $(22.0 \%)$ and Canada $(20.0 \%)$ (see figure 1$)$.

\section{Patient and public involvement}

APrON cohort participants were not involved in the development of the initial study design or in the recruitment of participants. However, APrON established a parent advisory committee (PAC) shortly after recruitment ended in 2012. Over time, 70 parents have participated on the PAC, with approximately 10-15 parents taking part in twice yearly meetings to advise the principal and co-investigators on a variety of topics, for example, how to keep participants engaged and interested in APrON research, priorities for future APrON research, and feedback on laymen research summaries posted to APrON's website. APrON has also extensively disseminated knowledge to the public via various modalities including: multiple presentations to various stakeholders, APrON's Newsletter, website (https://apronstudy.ca/) and conferences. Held via Zoom due to COVID-19 on 29-30 October 2020, APrON hosted an online conference titled Celebrating 10 Years: Alberta Pregnancy Outcomes and Nutrition (APrON) Achievements E Evolution, that attracted over 300 attendees, many who were APrON participants. The APrON cohort PAC members held their own panel session where they spoke to the attendees about their experiences with APrON, why they think the research is important, and how we can continue for years to come.

\section{How often have APrON families been followed up?}

Between 2009 and 2012, the initial cohort completed questionnaires and clinical assessments three times during pregnancy, and at 3 months postpartum. Hospital delivery records were also accessed to record birth information. Follow-up questionnaires were provided when children were 6 months, and 1, 2, 3, 5 and 8 years of age (see figure 2). We plan a 12-year follow-up beginning in 2022.

In total, 2189 women and 1325 men completed at least one questionnaire. Of the women and their children $(88.9 \%)$ are continuing participants, defined as those who did not become ineligible or were lost to follow-up. Participants who discontinued the study included women who miscarried, had a stillbirth, lost custody of their child or for whom a maternal/child death was reported $(n=52,2.4 \%)$; moved out of Calgary or Edmonton before 3 months postpartum $(n=43,2.0 \%)$ or withdrew from the study citing loss of interest $(\mathrm{n}=149,6.8 \%)$. The demographic characteristics of participants who continued in APrON compared with those that discontinued are provided in table 2 .

Continuing participants were more likely to be older, married and to have higher education and household incomes (all p's $<0.05$ ). The proportion of continuing participants born in Canada was similar to discontinuing participants. When mothers were lost to follow-up, so too were fathers and children.

\section{WHAT HAS THE APRON STUDY MEASURED?}

Expectant women and later mothers were asked to complete questionnaires three times during pregnancy 
Table 1 Participant intake characteristics $(n=3514)$

\section{Category}

Maternal (n/\%)

Paternal (n/\%)

Demographics

Age (years)

$\begin{array}{llr}16-24 & 100(4.6) & 19(1.4) \\ 25-29 & 481(22.0) & 216(15.4) \\ 30-34 & 980(44.8 \%) & 493(35.2) \\ 35-39 & 512(23.4) & 329(23.5) \\ \geq 40 & 105(4.8) & 162(11.6) \\ \text { Missing age information } & 11(0.5) & 181(12.9)\end{array}$

Marital status

$\begin{array}{lcc}\text { Married/common-law } & 2035(93.0) & 1275(91.1) \\ \text { Single/divorced/separated } & 97(4.4) & 27(1.9) \\ \text { Missing marital information } & 57(2.6) & 98(7.0)\end{array}$

Education

Less than a high school diploma

$59(2.7)$

$31(2.2)$

Completed high school diploma

$202(9.2)$

147 (10.5)

Completed trade, technical

$404(18.5)$

$376(26.9)$

Completed university or more

1442 (66.8)

746 (53.3)

Missing education information

$83(3.7)$

$100(7.1)$

Ethnicity

\begin{tabular}{|c|c|c|}
\hline White & $1693(77.3)$ & $1082(77.3)$ \\
\hline Non-white & $485(22.2)$ & $206(14.7)$ \\
\hline Missing ethnic information & $11(0.5)$ & $112(8.0)$ \\
\hline$<\$ 40000$ & $190(8.7)$ & - \\
\hline$\$ 40000-\$ 69999$ & $279(12.7)$ & - \\
\hline$>\$ 100000$ & $1146(52.3)$ & - \\
\hline Missing household income information & $110(5.0)$ & - \\
\hline \multicolumn{3}{|l|}{ Born in Canada } \\
\hline Yes & $1618(73.9)$ & $1024(73.1)$ \\
\hline No & $481(22.0)$ & $277(19.8)$ \\
\hline Primigravida & $996(46.4)$ & $\mathrm{n} / \mathrm{a}$ \\
\hline Missing gravidity information & $42(2.0)$ & $\mathrm{n} / \mathrm{a}$ \\
\hline \multicolumn{3}{|l|}{ Parity } \\
\hline 0 & $1222(55.8)$ & $\mathrm{n} / \mathrm{a}$ \\
\hline 1 & $732(33.4)$ & $\mathrm{n} / \mathrm{a}$ \\
\hline 2 & $181(8.3)$ & $\mathrm{n} / \mathrm{a}$ \\
\hline$\geq 3$ & $37(1.7)$ & $\mathrm{n} / \mathrm{a}$ \\
\hline Missing parity information & $17(0.8)$ & $\mathrm{n} / \mathrm{a}$ \\
\hline
\end{tabular}

$(<14,14-26$ and 27-40 weeks gestation; number of scales administered was 37) and seven times postnatally (3 and 6 months, 1, 2, 3, 5 and 8 years; number of scales administered was 77). Their partners (usually self-reported fathers) were asked to complete questionnaires at 14-26 weeks gestational age (number of scales 


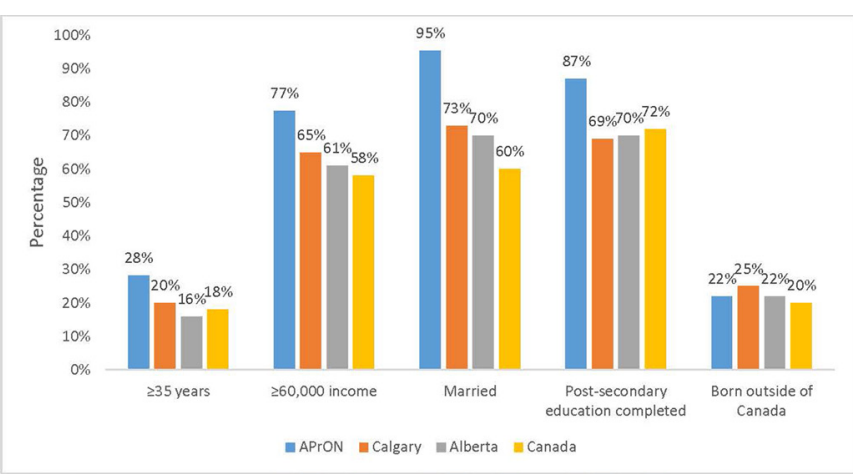

Figure 1 Sociodemographic characteristics of women in APrON, Calgary, Alberta and Canada.

administered was 16) and two times postnatally (3 months and 5 years; number of scales administered was 14). Questionnaires administered during pregnancy measured previous and current mental and physical health status, nutritional, medication and supplement intake, lifestyle choices, healthcare services used, social support, attitudes towards breast feeding and sociodemographic variables. The follow-up postpartum questionnaires collected information about labour and delivery, postpartum medical problems, maternal and paternal mental health and stress, nutrition, medication and supplement intake, lifestyle choices, food security, parenting, experiences in close relationships, adverse childhood experiences, and sociodemographics (see figure 2). Measures were selected based on several synergistic priorities. First, the APrON team had to judge measures to be reliable and valid indicators of the constructs of interest. Second, measures had to minimise burden to participants in terms of time and difficulty to complete, for example, measures with fewer items and more accessible language were valued most. Finally, an APrON team member had to express an interest in using the measure. Full details and documentation of all APrON measures are available on request.
Clinic visits were undertaken to gather maternal anthropometric measurements during pregnancy and at 3 months postpartum. These measures included height, pre-pregnancy and current weight, highest weight during pregnancy, circumference measurements (i.e., mid-upper arm, waist, hip, thigh) and skinfold thickness (i.e., biceps, triceps, subscapular, suprailiac, thigh). Each measurement was taken three times and averages were reported for analyses. Paternal height and weight were collected during the prenatal period. As an objective of APrON was to establish a biobank for future research on genetics, epigenetics, ${ }^{1}$ nutrient exposures and neurotoxicants, biosamples were also collected from mothers, their partners and infants during clinic visits. Midstream random urine samples $(10 \mathrm{~mL})$ were collected from expectant mothers at 14-26 weeks gestation and 3 months postpartum. A breast milk sample was collected from breastfeeding mothers to determine fatty acid composition. Maternal non-fasting venous blood samples were collected from mothers at $<14,14-26$ and 27-40 weeks gestation, and at 3 months postpartum. Maternal blood collection provided whole blood, plasma and serum, which were used for DNA, RNA, cytokines, haematocrit, haemoglobin measures, thyroid hormones and measures of heavy metals (eg, mercury, lead) and perfluorinated chemical exposure. Buccal cell samples were also collected at 14-26 weeks gestation from partners for DNA extraction and future genetic and epigenetic studies. From infants, buccal and/or blood samples were collected at 3 months of age for DNA extraction. Purified DNA is available at the Gene Expression Omnibus data repository (https://www.ncbi.nlm.nih.gov/geo/). A detailed list of data collected up to 8 years of age on the full sample is provided in table 3 for mothers, table 4 for fathers and table 5 for children.

Numerous substudies, employing portions of the APrON sample, have utilized the originally collected biosamples in related research; for example, the Neurotoxicant



Figure 2 Data collection to 8 years of child age. 
Table 2 Comparison of demographic characteristics of continuing and discontinued participants

Continuing

participants

Discontinuing participants

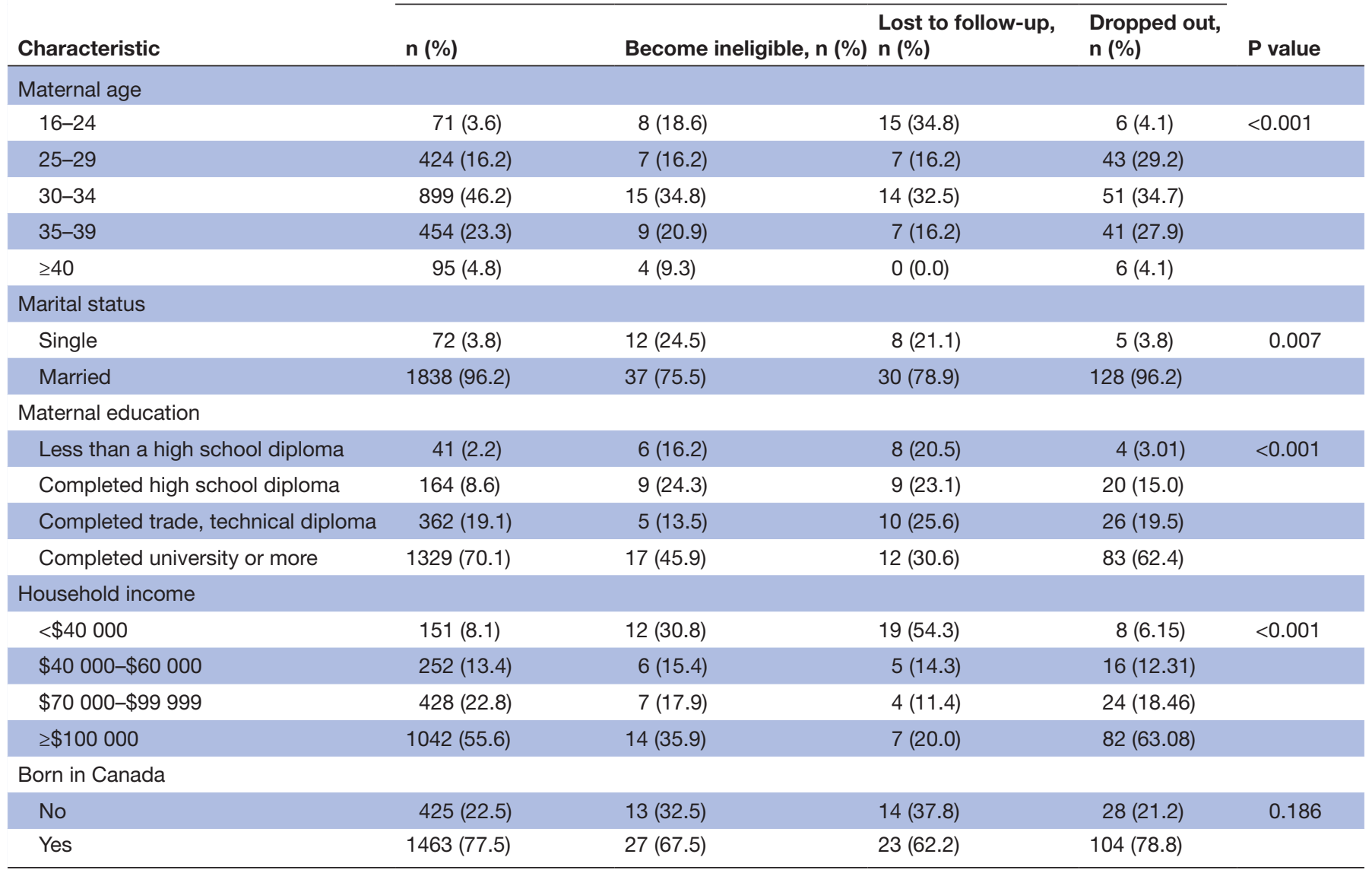

Assessing the null hypothesis that there is no difference in distributions between those participants who continued and those who did not continue in the study; $\chi^{2}$ test.

Study ( $\mathrm{n}=546)$ that examined maternal exposure to endocrine hormone disrupting chemicals (i.e., bisphenol A and phthalates) on child outcomes ${ }^{8-13}$ and the Parenting Research on Mental Illness, Stress and Epi/genetics (PROMISE; $\mathrm{n}=276$ ) Study of gene-by-environment interactions. ${ }^{14-18}{ }^{19-26}$ Another substudy, called the Fetal Programming Study ${ }^{19-27}(\mathrm{n}=276)$ collected additional data such as maternal and infant saliva for cortisol assessment, infant heart rate variability, and parent-infant interactions via videotaped observations.

\section{WHAT HAS APRON FOUND?}

A complete list of publications from APrON can be found at http://www.apronstudy.ca. Key findings from the full sample on maternal and paternal mental health, maternal nutrition and child outcomes have been summarised in box 1 .

\section{MENTAL HEALTH OUTCOMES}

Drawn from the full APrON cohort, three key papers ${ }^{28-30}$ reported on perinatal depression and anxiety symptom prevalence and risk factors and specifically, the impact of adverse childhood experiences on outcomes. Many more papers report on parental mental health in substudies. See APrONstudy.ca for details.

\section{Predictors of depression}

Maternal prenatal depression is known to have negative impacts on pregnancy and maternal postnatal mental health and neonatal outcomes, ${ }^{31}{ }^{32}$ and the psychosocial predictors of maternal postpartum depression are well known. ${ }^{33} 34$ However, less well known is whether mothers and fathers in partnered, cohabitating couples (e.g., married, common-law) share common predictors and experiences of depressive symptoms in the perinatal period. Further, whether maternal prenatal nutrition impacts postpartum depressive symptoms is less well studied. Thus, using APrON data, Leung and colleagues $^{30}$ reported on the prevalence of depressive symptoms in couples. For partnered mothers' depressive symptoms, using the recommended Edinburgh Depression Scale (EDS) cut-off score of $\geq 10,{ }^{35}$ findings revealed that $15.2 \%$ and $11.18 \%$ of mothers had significant symptoms prenatally and at 3 months postpartum, respectively. For fathers in couples, the cut-off of EDS $\geq 9$ was used as 


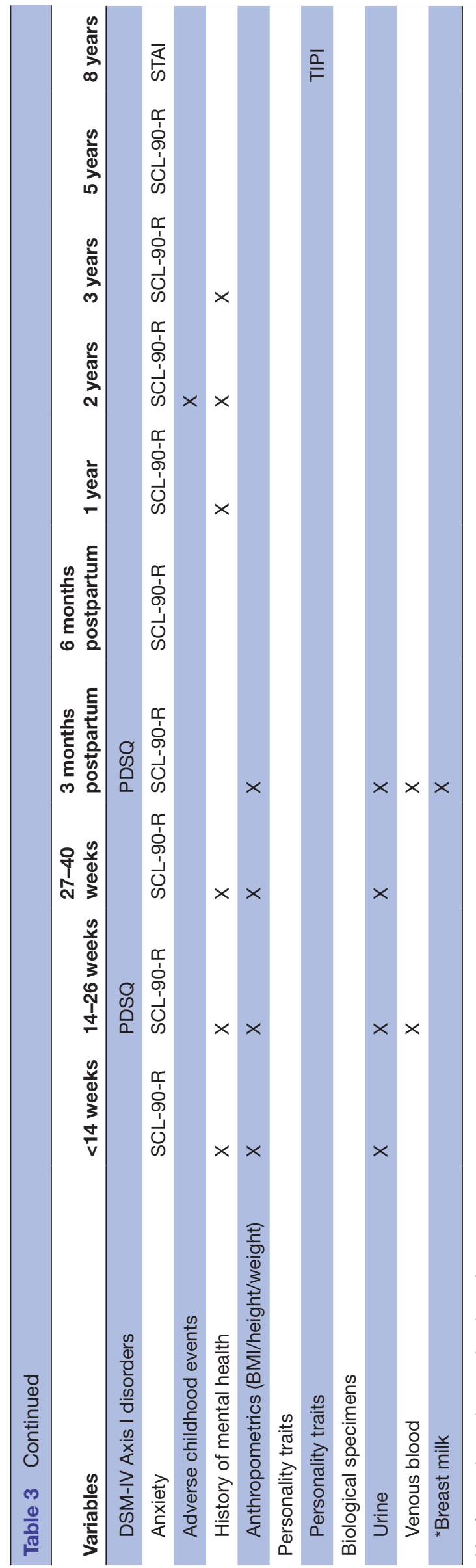

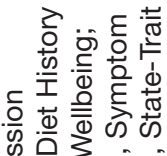

क्षे

产要客的

क

क क के

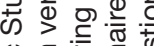

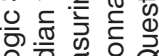

은

हิ

은

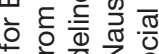

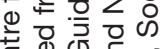

ब

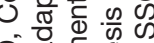

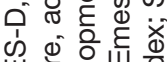

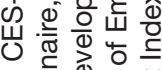

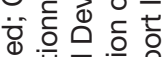

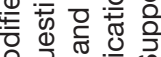

으을

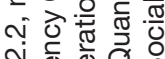

N क्ष

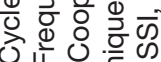

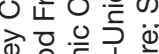

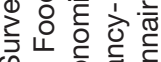

के टठ ฮ

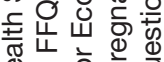

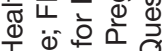

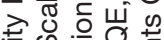

C

है 둥

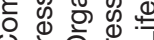

당

元

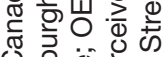

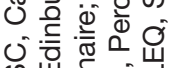

की

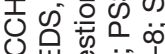

बे

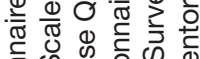

을 은 든

क क

응효

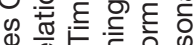

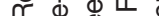

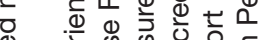

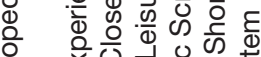

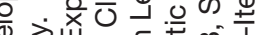

这山.

\% 000 \%

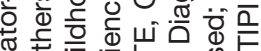

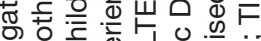

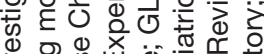

ه

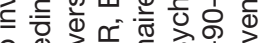

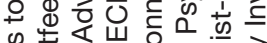

क्ष कि के

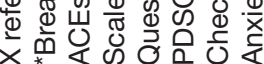


Table 4 Paternal variables and biological specimens collected via questionnaires and clinic visits

\begin{tabular}{|c|c|c|c|c|c|}
\hline Variables & $<14$ weeks gestation & 14-26 weeks & $\begin{array}{l}27-40 \\
\text { weeks }\end{array}$ & $\begin{array}{l}3 \text { months } \\
\text { postpartum }\end{array}$ & $\begin{array}{l}5 \text { years } \\
\text { postpartum }\end{array}$ \\
\hline \multicolumn{6}{|l|}{ Sociodemographics } \\
\hline Education & & & $x$ & & \\
\hline Marital status & & & $x$ & & \\
\hline Born in Canada & & & $x$ & & \\
\hline Family background & & & $x$ & & $x$ \\
\hline Family history & & & $x$ & & \\
\hline \multicolumn{6}{|l|}{ Health and lifestyle } \\
\hline Substance use & & $\mathrm{X}$ & $x$ & $X$ & $x$ \\
\hline \multirow{2}{*}{$\begin{array}{l}\text { Physical activity anthropometrics } \\
\text { (BMl/height/weight) }\end{array}$} & & GLTE & GLTE & GLTE & GLTE \\
\hline & & $x$ & $x$ & $x$ & \\
\hline \multicolumn{6}{|l|}{ Psychosocial health } \\
\hline Depression & & EDS & EDS & EDS & EDS \\
\hline Social support & & SSQ & SSQ & SSQ & SSQ \\
\hline Stress & & & SLEQ & & SLEQ \\
\hline Anxiety & & & & & SCL-90-R \\
\hline
\end{tabular}

$X$ refers to investigator-developed measures.

BMI, body mass index; EDS, Edinburgh Depression Scale; GLTE, Godin Leisure-Time Exercise Questionnaire; SLEQ, Stressful Life Events Questionnaire; SSQ, Social Support Questionnaire.

per recommendations ${ }^{36}$ revealing that $14.0 \%$ and $12.1 \%$ of fathers had significant symptoms prenatally and at 3 months postpartum, respectively. These rates are consistent with other large samples. ${ }^{3738}$ Further, $2.3 \%$ of couples experienced perinatal depressive symptoms in both mothers and fathers, $78.5 \%$ of couples did not experience depressive symptoms in either partner, $9.5 \%$ of couples experienced depressive symptoms in mothers only and $9.8 \%$ of couples experienced depressive symptoms in fathers only. Leung and colleagues ${ }^{30}$ also determined the predictors of depression at 3 months postpartum from second trimester data. Risk factors for maternal postpartum depressive symptoms included low household income, high prenatal depressive symptoms, low postnatal support from fathers and high stressful life events. Risk factors for paternal postpartum depressive symptoms included low household income, prenatal depressive symptoms, postpartum stressful life events and smoking. For couples in which both partners were depressed, risk factors included low income, high maternal depressive symptoms and low prenatal social support from fathers. Postpartum social support was found to be a protective factor associated with decreased risk of maternal and paternal postpartum depressive symptoms.

Additionally, Leung and colleagues ${ }^{28}$ examined nutritional predictors of maternal postpartum depression, focusing on APrON mothers' reports of their multivitamin and mineral intake in each trimester of pregnancy. Almost all women (99\%) took some type of micronutrient supplement during the prenatal period, with the most commonly consumed being vitamins B6, B9 (folate), B12 and E, with more than $90 \%$ of mothers consuming above recommended dietary allowances. With respect to predictors of postpartum depression, findings revealed that mothers who experienced postpartum depressive symptoms (EDS $\geq 10$ ) were statistically less likely to take the supplemental micronutrients selenium and omega-3, compared with mothers who reported no postpartum depressive symptoms (EDS $<10)$. Selenium intake above the recommended dietary allowance appeared to be 
Table 5 Child variables and biological specimens collected in questionnaires and clinic visits

\begin{tabular}{|c|c|c|c|c|c|c|c|}
\hline Variables & 3 months & 6 months & 1 year & 2 years & 3 years & 5 years & 8 years \\
\hline Health and development & & $x$ & $x$ & $x$ & $x$ & $\mathrm{X}$ & $x$ \\
\hline Occupation & $\mathrm{X}$ & $X$ & $\mathrm{X}$ & $\mathrm{X}$ & $\mathrm{X}$ & $\mathrm{X}$ & \\
\hline Childcare & $\mathrm{X}$ & $\mathrm{x}$ & $x$ & $\mathrm{X}$ & $x$ & $x$ & \\
\hline Vaccines & & & & & & $\mathrm{X}$ & \\
\hline Child food and liquid intake & $\mathrm{X}$ & $\mathrm{x}$ & $\mathrm{X}$ & $\mathrm{X}$ & $x$ & $\mathrm{X}$ & \\
\hline Children's eating and drinking behaviours & & & & & & $\mathrm{X}$ & $x$ \\
\hline Children's activities & & & & & & $\mathrm{X}$ & $x$ \\
\hline Children's injuries & & & & & & $\mathrm{X}$ & \\
\hline Community & & & & & & & $x$ \\
\hline Children's education & & & & & & & $x$ \\
\hline \multicolumn{8}{|l|}{ General infant behaviours } \\
\hline Infant Behavior Questionnaire (IBQ) & $\mathrm{x}$ & $\mathrm{x}$ & $\mathrm{x}$ & $\mathrm{x}$ & & & \\
\hline Scales of Independent Behaviors (SIB-R) & $x$ & $x$ & $x$ & $x$ & $x$ & $x$ & \\
\hline Fussing and crying & $\mathrm{X}$ & $\mathrm{x}$ & $\mathrm{X}$ & $\mathrm{X}$ & & & \\
\hline Sleeping & & $x$ & $x$ & $x$ & $x$ & $x$ & $x$ \\
\hline Early Childhood Behavior Questionnaire (ECBQ) & & & & $\mathrm{x}$ & & & \\
\hline Children's Behavior Questionnaire (CBQ) & & & & & $x$ & & \\
\hline Child Behaviour Checklist Ages 1.5-5 (CBCL) & & & & $\mathrm{X}$ & $x$ & & \\
\hline $\begin{array}{l}\text { Behaviour Rating Inventory of Executive Function- } \\
\text { Preschool (BRIEF-P) }\end{array}$ & & & & $x$ & $x$ & & \\
\hline $\begin{array}{l}\text { Ages and Stages Questionnaire-Third Edition } \\
\text { (ASQ-3) }\end{array}$ & & & & & & $\mathrm{x}$ & \\
\hline $\begin{array}{l}\text { Children's Communication Checklist-Second } \\
\text { Edition (CCC-Q) }\end{array}$ & & & & & & $x$ & \\
\hline $\begin{array}{l}\text { Behaviour Assessment System for Children- } \\
\text { Second Edition (BASC-2) }\end{array}$ & & & & & & $x$ & $\mathrm{X}$ \\
\hline Media use & & & & & & $x$ & $x$ \\
\hline Quantitative Checklist for Autism in Toddlers & & & & $\mathrm{X}$ & $x$ & & \\
\hline Temper Tantrum Scale-past 2 weeks & & & & $x$ & $x$ & & \\
\hline Temper Tantrum Scale-past 6 months & & & & $\mathrm{x}$ & $\mathrm{x}$ & & \\
\hline Parenting & & & & & & $x$ & $x$ \\
\hline \multicolumn{8}{|l|}{ Biological specimens } \\
\hline Buccal cells & $\mathrm{X}$ & & & & & & \\
\hline Venous blood & $\mathrm{X}$ & & & & & & \\
\hline
\end{tabular}

protective, and other research suggests that this micronutrient has a role in normal brain function and in the pathway between dopamine and the pathophysiology of depression. ${ }^{39}$ The supplement taken the least was omega-3; however, $68.5 \%$ of women with low depressive symptoms $(\mathrm{EDS}<10)$ did not take omega-3, versus $78.0 \%$ of women with high depressive symptoms (EDS $\geq 10$ ), a finding consistent with other research. ${ }^{40}$ The mean intakes of other nutrients were more likely to be higher in women with low depressive symptoms than those with high symptoms, although not statistically significant. The authors conclude with a call for more study into the value of selenium and omega-3 in the prevention of postpartum depression.

\section{Associations with adverse childhood experiences}

Adverse childhood experiences are defined as a set of exposures to personal abuse, neglect and household dysfunction prior 18 years of age that includes physical, sexual and emotional abuse, domestic violence, and parental mental illness, substance use and incarceration. ${ }^{41}$ A graded relationship has been repeatedly observed between the number of adversities experienced in childhood and later increased risk for poor health, including depression ${ }^{42}{ }^{43}$; however, whether such adversities affect perinatal mood more broadly including anxiety was less studied. Felitti and colleagues ${ }^{41}$ classic measure of adverse childhood experiences was administered to APrON mothers, revealing that while $55 \%$ reported 


\section{Box 1 Key findings}

\section{Mental health outcomes}

- In partnered couples, both mothers and fathers had depressive symptoms in $2.3 \%$ of couples. Low household income and prenatal maternal depression were associated with a higher probability of depressive symptoms in both partners. In $78.5 \%$ of couples, neither father nor mother experienced depressive symptoms. Postnatal social support was found to be a protective factor associated with decreased risk of maternal and paternal postpartum depressive symptoms. Risk factors for maternal postpartum depression symptoms included low household income, and prenatal symptoms of depression. For paternal postpartum depression symptoms, risk factors included low household income, prenatal depression symptoms, postpartum stressful life events, and smoking.

- Women with higher postpartum depression symptoms were less likely to take micronutrients (eg, vitamins B6, B9, B12 and E; essential fatty acids; selenium) compared to those with fewer symptoms; however, only selenium and omega-3 were significantly different between symptomatically depressed and non-depressed groups, with non-depressed mothers consuming higher amounts.

- $13 \%$ of mothers had a score of three or more adverse childhood experiences. Maternal exposure to early adversities predicted higher reported symptoms of perinatal depression and anxiety.

\section{Nutrition outcomes}

- Approximately $70 \%$ of women who entered pregnancy with a body mass index $(\mathrm{BMI})>25 \mathrm{~kg} / \mathrm{m}^{2}$ were likely to exceed gestational weight gain guidelines in the beginning of the second trimester. Women with excessive weight gain also gained higher amounts of body fat and retained higher amounts of fat compared with women who gained within the guidelines. Women who consumed a healthy diet pattern prior to pregnancy were less likely to develop complications such as gestational hypertension.

- The median vitamin D intake from diet and supplements was $600 \mathrm{IU} /$ day during pregnancy, which was not enough to achieve a target plasma circulation $25(\mathrm{OH}) \mathrm{D}$ concentration. A significant relationship between maternal reported dietary vitamin $D$ intake and plasma 25(OH)D and 3-epi-25(OH)D3 concentration were identified.

- Only $23 \%$ of mothers met the adequate intake (Al) recommendations for choline; the number was even lower $(10 \%)$ in the postpartum period. Consuming eggs and milk during pregnancy increased mothers' likelihood of meeting choline Al recommendations.

\section{Child outcomes}

- Infants with higher birth weights and at 3 months of age had mothers with a higher pre-pregnancy BMI. Gestational weight gain above recommendations was associated with higher infant weight at birth and 3 months of age as well as more rapid postnatal growth.

- Infants' vitamin D status increases in direct proportion to mothers' vitamin $\mathrm{D}$ intake. Given many mothers were found to be below recommended Al for vitamin D, both infants and breastfeeding mothers require vitamin $\mathrm{D}$ supplementation.

- Maternal experiences of early adversity associate with maternal symptoms of anxiety and depression during the perinatal period as well as externalising behavioural problems in their 2-year old children. Boys demonstrate greater vulnerability to the indirect effects of maternal adversity via both depression and anxiety.

- Both mothers' perinatal depressive symptoms and mothers' and fathers' co-occurring perinatal depressive symptoms predicted more problematic emotionally reactive, withdrawn, and total internalising behaviours in their 2-3-year old children. Children's aggression,

Continued
Box 1 Continued

attention problems and total externalising behaviours were only predicted by mothers' perinatal depressive symptoms.

no early adverse childhood experiences, nearly $32 \%$ reported one or two adversities and $13 \%$ reported three or more. Employing data from each trimester of pregnancy and 3, 6 and 12 months postpartum, more reported early adversities predicted higher reported symptoms of prenatal and postnatal depression and anxiety. ${ }^{29}$ Routine screening for maternal childhood adversities may facilitate early identification and preventative interventions for perinatal mood disorders including depression and anxiety; however, this has been recently debated. ${ }^{43}$ In sum, the identified risks and protective factors provide opportunities for prevention of perinatal depression and anxiety.

\section{NUTRITION OUTCOMES}

Gestational weight gain and dietary intake, especially perinatal nutrient intake and status focused on vitamin D and choline were examined in eight key papers. ${ }^{44-51}$

\section{Gestational weight gain and dietary intake}

Gestational weight gain over or below Health Canada's Gestational Weight Gain Guidelines is associated with an increased risk of adverse outcomes for mother and infant. ${ }^{52}$ The APrON cohort was the first to provide evidence that more than half of women gain more than Health Canada's Guidelines, using prospectively collected body weights from across pregnancy. ${ }^{44}$ Since then, several studies have been completed with body weight or body composition in pregnancy or postpartum as a main outcome. Analysis of the APrON data by Jarman and colleagues ${ }^{45}$ provided a clear picture indicating that approximately $70 \%$ of women who become pregnant with a body mass index (BMI) $>25 \mathrm{~kg} / \mathrm{m}^{2}$ are likely to exceed gestational weight gain guidelines, by about 19 weeks gestation. Women with excessive weight gain also gain higher amounts of body fat and retain higher amounts of fat compared with women who gain within the guidelines. ${ }^{46}$ The detailed dietary intake information that was collected showed that women who consumed a healthy diet pattern prior to pregnancy were less likely to develop complications such as gestational hypertension. ${ }^{47} \mathrm{An}$ individualised approach to supportive dietary counselling that considers pre-pregnancy BMI in addition to the woman's social and financial context may be key to helping women meet national recommendations for both diet and gestational weigh gain in pregnancy. ${ }^{53}$

\section{Nutrient intake and status}

Vitamin D is critical for a healthy pregnancy and the major circulating form of vitamin $\mathrm{D}$ is 25-hydroxyvitamin D $(25(\mathrm{OH}) \mathrm{D})$; thus, the total serum $25(\mathrm{OH}) \mathrm{D}$ level is 
Table 6 Response rates for questionnaires

\begin{tabular}{llll}
\hline Data collection time point & Targeted participants (n) & Participants responded (n) & Response rate (\%) \\
\hline $\begin{array}{l}\text { Mothers <14 weeks gestation } \\
\text { Mothers at 14-26 weeks gestation }\end{array}$ & 2189 & 2145 & 97.9 \\
$\begin{array}{l}\text { Mothers at 14-26 weeks gestation and } \\
\text { those recruited at <14 weeks gestation }\end{array}$ & 563 & 475 & 84.3 \\
Fathers at 14-26 weeks gestation & 1325 & 1315 & 99.2 \\
\hline Mothers at 27-40 weeks gestation & 1838 & 1830 & 99.5 \\
Mothers at 3 months postpartum & 2051 & 1811 & 88.3 \\
\hline Fathers at 3 months postpartum & 1287 & 1150 & 89.3 \\
\hline Mothers at 6 months postpartum & 1727 & 1523 & 88.1 \\
\hline Mothers at 1 year postpartum & 1747 & 1305 & 74.7 \\
\hline Mothers at 2 years postpartum & 1809 & 1299 & 71.8 \\
\hline Mothers at 3 years postpartum & 1648 & 1282 & 77.7 \\
\hline
\end{tabular}

currently considered the best indicator of vitamin D supply. ${ }^{54}$ Three studies that included APrON participants examined the role of maternal vitamin D intake during pregnancy. ${ }^{48-50}$ Aghajafari and colleagues ${ }^{49}$ reported that the median vitamin $\mathrm{D}$ intake from diet and supplements was 600 International Units (IU)/day during pregnancy, which was not sufficient to achieve a target circulation of $25(\mathrm{OH}) \mathrm{D})$ concentration. When the 3-epimer (3-epi-25(OH)D3) was included in the estimation of vitamin D status, the prevalence of vitamin D insufficiency $(<75 \mathrm{nmol} / \mathrm{L})$ was lower $(33.0 \%)$ compared with when it was excluded $(38.0 \%)$. Vitamin D supplementation (2000 IU/day) was associated with $25(\mathrm{OH}) \mathrm{D} 3$ sufficiency. ${ }^{48-50}$ A significant relationship between maternal reported dietary vitamin $\mathrm{D}$ intake and plasma $25(\mathrm{OH}) \mathrm{D}$ and 3-epi-25(OH)D3 concentration was identified.

Choline intake during pregnancy and lactation is critical to the offspring's brain function. ${ }^{55}$ Lewis and colleagues $^{51}$ reported that the mean choline intake in pregnant and lactating women ranged between 340 (SD 148 ) in the second trimester and 346 (SD 151) $\mathrm{mg} /$ day at 3 months postpartum. Only $23 \%$ of mothers met the adequate intake (AI) recommendations for choline; the number was even lower $(10 \%)$ in the postpartum period. ${ }^{51}$ Consuming eggs and milk during pregnancy increased mothers' likelihood of meeting choline AI recommendations. These findings contributed to the European Food Safety Authority dietary reference values for choline guidelines. ${ }^{56}$ To conclude, a significant number of women were not meeting recommendations for vitamin D and choline intake during pregnancy and postpartum. Increased consumption of these nutrients may be necessary for pregnant and lactating women to improve the health and development of their infants.

\section{CHILD OUTCOMES}

The impacts of maternal and infant nutrient intake, maternal BMI and gestational weight gain, and perinatal depression and maternal adverse childhood experiences on child outcomes were examined in four key papers. ${ }^{29}$ M7-59 Many more papers will emerge as the cohort matures and data become available.

\section{Maternal BMI, gestational weight gain and vitamin D}

Subhan and colleagues ${ }^{57}$ described the effects of maternal pre-pregnancy BMI and gestational weight gain on infant anthropometrics at birth and 3 months and infant growth rates between birth and 3 months. Other research shows that children who are heavier at birth have an increased risk of high BMI in childhood, being overweight or obese as adults, ${ }^{6061}$ a condition linked to increased risk for hypertension, type 2 diabetes and cardiovascular diseases. ${ }^{60} 62$ APrON findings revealed that infants with higher weight at birth and at 3 months of age had mothers with a higher pre-pregnancy BMI. Also gestational weight gain above recommendations was associated with higher infant weight at birth and 3 months of age as well as more rapid postnatal growth. The authors concluded that clinicians and healthcare professionals should encourage women to enter pregnancy with a healthy BMI and adhere to the current gestational weight gain recommendations.

Aghajafari and colleagues ${ }^{58}$ examined the association between APrON mothers' vitamin D intake during breast feeding with their infants' vitamin D status to determine whether infant supplementation was sufficient. Other research reveals that low vitamin D is associated with increased rates of infections, autoimmunity and allergies $^{63}$ and poor bone health in children. ${ }^{64}$ Using plasma from a subset of breastfed infants, vitamin D status was measured by liquid chromatography-tandem mass spectrometry and mothers' and infants' dietary data were attained from questionnaires. Controlling for race, season, prenatal maternal vitamin $\mathrm{D}$ status, infant birth weight and daily infant vitamin $\mathrm{D}$ supplementation, infants' vitamin D status increased in direct proportion to mothers' vitamin D intake. Moreover, a quarter of mothers were below recommended AI for vitamin $\mathrm{D}$. The authors concluded that to ensure optimal infant vitamin 
D status, both infants and breastfeeding mothers require vitamin D supplementation.

\section{Perinatal depression and adverse childhood experiences}

Two studies addressed impacts of perinatal depression and adverse childhood experiences on children's behaviour. It is well established that children's internalising behavioural problems (e.g., emotional reactivity, anxiety, depression, somatic complaints and social withdrawal) and externalising behavioural problems (i.e., inattention, hyperactivity and aggression) predict adult mental health problems. ${ }^{65-67}$ As indicated above, adverse childhood experiences increased risk for poor health over the lifespan ${ }^{68-70}$; however, intergenerational impacts have also been noted ${ }^{71} 72$ with an incomplete understanding of the mechanism of transmission from one generation to the next. ${ }^{29}$ In examining intergenerational impacts, Letourneau and colleagues ${ }^{29}$ found that APrON mothers' experiences of early adversity were associated with maternal symptoms of anxiety and depression during the perinatal period (discussed earlier) as well as externalising behavioural problems in their 2-year old children. Indirect associations were observed between maternal adverse childhood experiences and children's internalising and externalising problems via maternal anxiety and depression. Sex differences were also observed with boys demonstrating greater vulnerability to the indirect effects of maternal adversity via both depression and anxiety. The authors concluded that interventions may be targeted to women who have multiple adverse childhood experiences as well as mental health problems to prevent behavioural problems in their children. Letourneau and colleagues ${ }^{59}$ further examined perinatal depression in mothers, as well as fathers or both parents and how symptoms in one or both parents predict children's behavioural problems. Other research has shown that fathers may buffer the impacts of maternal depression on children's outcomes and thus this protective factor is likely undermined when fathers are depressed as well as mothers. ${ }^{32}{ }^{73}$ Mothers' and fathers' depressive symptoms were measured during pregnancy and 3 months postpartum, and children's behaviour was measured at 24 and 36 months of age. Results revealed that both mothers' perinatal depressive symptoms and mothers' and fathers' co-occurring perinatal depressive symptoms predicted more problematic emotionally reactive, withdrawn and total internalising behaviours. In contrast, children's aggression, attention problems and total externalising behaviours were only predicted by mothers' perinatal depressive symptoms. The authors urge healthcare providers to consider the whole family, including fathers, when treating maternal symptoms of depression, aligned with review recommendations to also consider fathers. ${ }^{32} 74$

\section{WHAT ARE THE MAIN STRENGTHS AND WEAKNESSES?}

The major strengths of APrON are recruitment and retention of the large community-based cohort during pregnancy, postpartum and early childhood. The APrON study used a detailed array of standardised validated psychological and physiological measures and biospecimens. The prospective data collection and follow-up enabled investigation of a wide range of maternal and paternal health outcomes. The APrON team has been successful in continuously engaging participants, as evidenced by high response rates over the years (see table 6).

The high response rates over time may be due to the recruitment of a relatively low-risk sample as sociodemographic characteristics are skewed toward a high percentage of well-educated, higher income, married participants. Consequently, generalisability of findings to those with different sociodemographic backgrounds should be made with caution. Further, the study likely included participants with healthier lifestyle behaviours or with more positive attitudes towards research, because such characteristics are associated with higher socioeconomic status. ${ }^{75}$ Selective attrition bias is likely operating in the cohort, which may lead to underestimation of the effect of parental mental health and lifestyle choices on children's development. While attrition has been relatively low over time, differences in the sociodemographic characteristics of the continuing participants compared with discontinuing participants should be considered when interpreting the findings. There were also fewer fathers than mothers in the sample; thus, most developmental and behavioural outcomes are based on maternal reports.

\section{WHERE CAN I FIND OUT MORE ABOUT APRON RESEARCH?}

Information about the APrON study is available at www.apronstudy.ca. APrON data are available through

\section{Profile in a nutshell}

The APrON study is a longitudinal cohort of mothers, fathers and their children investigating mental health, nutrition, and child development outcomes.

- A total of 2189 women women (aged 16-44) and 1325 fathers (aged 18-52), residing around Calgary or Edmonton, Alberta, enrolled and completed at least one questionnaire between May 2009 and June 2012. The majority were married, educated, had annual household incomes $>\$ 70000$ and were born in Canada.

- Mothers completed questionnaires and clinic visits spanning pregnancy to 8 years postpartum and provided access to their medical records as well as blood and urine samples.

- The APrON dataset includes comprehensive maternal and child sociodemographic data, maternal nutrition and psychological data from pregnancy to 8 years postpartum, and infant and child neurodevelopmental and behavioural data to 8 years of age. Maternal, paternal and infant biosamples for genetics were also collected. Five-year and 8-year data collection is complete and 12-year data collection will start in 2022

Requests for data and collaboration are welcomed, contact Dr. Nicole Letourneau at nicole.letourneau@ucalgary.ca 
Secondary Evidence to Generate Evidence (https:// policywise.com/sage/). For more information, contact Principal Investigator, Dr. Nicole Letourneau at Nicole. Letourneau@ucalgary.ca. Collaboration or data access inquiries will be considered by the APrON Study team.

\section{Author affiliations}

${ }^{1}$ Faculty of Nursing, University of Calgary, Calgary, Alberta, Canada

${ }^{2}$ Cumming School of Medicine (Departments of Paediatrics, Psychiatry \& Community Health Sciences), University of Calgary, Calgary, Alberta, Canada ${ }^{3}$ Cumming School of Medicine (Family Medicine \& Community Health Sciences), University of Calgary, Calgary, Alberta, Canada

${ }^{4}$ Faculty of Agricultural, Food and Nutritional Science, University of Alberta, Edmonton, Alberta, Canada

${ }^{5}$ Cumming School of Medicine (Pediatrics), University of Calgary, Calgary, Alberta, Canada

${ }^{6}$ Cumming School of Medicine (Pediatrics \& Community Health Sciences), University of Calgary, Calgary, Alberta, Canada

${ }^{7}$ Health Science - Public Health Program, University of Lethbridge, Lethbridge, Alberta, Canada

Acknowledgements The authors acknowledge the contributions of all the families who took part in the APrON study, and the investigators, managers, research assistants, graduate and undergraduate students, volunteers and clerical staff of the APrON study team. The team would also like to thank Dr. Elena Ali who led earlier drafts of this manuscript under the supervision of Drs. Nicole Letourneau and Gerald Giesbrecht.

Contributors NL conceived of the idea for the paper, oversaw all aspects of the APrON cohort, including the collection, cleaning and analysis of data for reviewed studies, and wrote major sections of the paper, approved the final version of the paper to be published and agreed to be accountable for all aspects of the work. FA contributed to the collection, cleaning and analysis of data for reviewed studies, critically reviewed the drafts, approved the final version of the paper to be published and agreed to be accountable for all aspects of the work. RCB wrote a key section of the paper, contributed to the collection, cleaning and analysis of data for reviewed studies, critically reviewed the drafts, approved the final version of the paper to be published and agreed to be accountable for all aspects of the work. AD contributed to the collection, cleaning and analysis of data for reviewed studies, critically reviewed the drafts, approved of the final version of the paper to be published and agreed to be accountable for all aspects of the work. DD contributed to the collection, cleaning and analysis of data for reviewed studies, critically reviewed the drafts, approved the final version of the paper to be published and agreed to be accountable for all aspects of the work. CF contributed to the collection, cleaning and analysis of data for reviewed studies, critically reviewed the drafts, approved the final version of the paper to be published and agreed to be accountable for all aspects of the work. GG contributed to the collection, cleaning and analysis of data for reviewed studies, critically reviewed the drafts, approved the final version of the paper to be published and agreed to be accountable for all aspects of the work. BK contributed to the collection, cleaning and analysis of data for reviewed studies, critically reviewed the drafts, approved the final version of the paper to be published and agreed to be accountable for all aspects of the work. BL contributed to the collection, cleaning and analysis of data for reviewed studies, critically reviewed the drafts, approved the final version of the paper to be published and agreed to be accountable for all aspects of the work. HN contributed to the cleaning and analysis of data for reviewed studies, critically reviewed the drafts, approved the final version of the paper to be published and agreed to be accountable for all aspects of the work. APrON Study Cohort Participants volunteered time and energy to share their individual and family's experiences with us to help improve the long-term health outcomes for future newborns, mother, fathers and families. NL is the author who is responsible for the overall content as the guarantor.

Funding This work was supported by the Alberta Children's Hospital Foundation (grant/award number N/A), Alberta Innovates Health Solutions Foundation (grant/ award number N/A), Canadian Institutes for Health Research (Operating Grants), Canadian Institutes for Health Research (CIHR Doctoral Award, grant number 394652), Kids Brain Health Network (grant/award number N/A) and the National Centre of Excellence: Allergen (grant/award number N/A).

Competing interests None declared.
Patient and public involvement Patients and/or the public were involved in the design, or conduct, or reporting, or dissemination plans of this research. Refer to the Methods section for further details.

Patient consent for publication Not applicable.

Ethics approval The APrON study was approved by the University of Calgary Health Research Ethics Board (REB14-1702) and University of Alberta Health Research Ethics Biomedical Panel (Pro00002954).

Provenance and peer review Not commissioned; externally peer reviewed.

Data availability statement Data are available upon reasonable request. Data may be obtained from a third party and are not publicly available. Data can be collected at the Secondary Analysis to General Evidence (SAGE) data repository or by contacting Dr Nicole Letourneau (the principal investigator for the APrON Study).

Open access This is an open access article distributed in accordance with the Creative Commons Attribution Non Commercial (CC BY-NC 4.0) license, which permits others to distribute, remix, adapt, build upon this work non-commercially, and license their derivative works on different terms, provided the original work is properly cited, appropriate credit is given, any changes made indicated, and the use is non-commercial. See: http://creativecommons.org/licenses/by-nc/4.0/.

\section{ORCID iDs}

Nicole Letourneau http://orcid.org/000-0002-7468-915x

Rhonda C Bell http://orcid.org/0000-0002-4298-9641

Bonnie Kaplan http://orcid.org/0000-0003-1911-0472

\section{REFERENCES}

1 Leung BMY, Giesbrecht GF, Letourneau N, et al. Perinatal nutrition in maternal mental health and child development: birth of a pregnancy cohort. Early Hum Dev 2016;93:1-7.

2 Kaplan BJ, Giesbrecht GF, Leung BMY, et al. The Alberta pregnancy outcomes and nutrition (APrON) cohort study: rationale and methods. Matern Child Nutr 2014;10:44-60.

3 Manca DP, O’Beirne M, Lightbody T, et al. The most effective strategy for recruiting a pregnancy cohort: a tale of two cities. BMC Pregnancy Childbirth 2013;13:1-7.

4 Manca DP, O'Beirne M, Lightbody T, et al. The most effective strategy for recruiting a pregnancy cohort: a tale of two cities. BMC Pregnancy Childbirth 2013;13:75.

5 Leung BM, McDonald SW, Kaplan BJ, et al. Comparison of sample characteristics in two pregnancy cohorts: community-based versus population-based recruitment methods. BMC Med Res Methodol 2013;13:149.

6 Dzakpasu S, Kaczorowski J, Chalmers B, et al. The Canadian maternity experiences survey: design and methods. J Obstet Gynaecol Can 2008;30:207-16.

7 Tough SC, McDonald SW, Collisson BA, et al. Cohort profile: the all our babies pregnancy cohort (AOB). Int $J$ Epidemiol 2017;46:1389-90.

8 England-Mason G, Liu J, Martin JW, et al. Postnatal BPA is associated with increasing executive function difficulties in preschool children. Pediatr Res 2021;89:1-8.

9 Liu J, Wattar N, Field CJ, et al. Exposure and dietary sources of bisphenol A (BPA) and BPA-alternatives among mothers in the APrON cohort study. Environ Int 2018;119:319-26.

10 Giesbrecht GF, Ejaredar M, Liu J, et al. Prenatal bisphenol a exposure and dysregulation of infant hypothalamic-pituitary-adrenal axis function: findings from the APrON cohort study. Environmental Health 2017;16:1-11.

11 Giesbrecht GF, Liu J, Ejaredar M, et al. Urinary bisphenol A is associated with dysregulation of HPA-axis function in pregnant women: findings from the APrON cohort study. Environ Res 2016;151:689-97.

12 Liu J, Martin LJ, Dinu I, et al. Interaction of prenatal bisphenols, maternal nutrients, and toxic metal exposures on neurodevelopment of 2-year-olds in the APrON cohort. Environ Int 2021;155:106601.

13 Grohs MN, Reynolds JE, Liu J, et al. Prenatal maternal and childhood bisphenol a exposure and brain structure and behavior of young children. Environmental Health 2019;18:1-12.

14 Letourneau NL, de Koning APJ, Sekhon B, et al. Parenting interacts with plasticity genes in predicting behavioral outcomes in preschoolers. Can J Nurs Res 2020;52:290-307.

15 Merrill SM, Gladish N, Fu MP, et al. Associations of peripheral blood DNA methylation and estimated monocyte proportion differences during infancy with toddler attachment style. Attach Hum Dev 2021:1-30. 
16 Merrill SM, Moore SR, Gladish N, et al. Paternal adverse childhood experiences: associations with infant DNA methylation. Dev Psychobiol 2021;63:e22174

17 Letourneau N, Ntanda H, Jong VL, et al. Prenatal maternal distress and immune cell epigenetic profiles at 3-months of age. Dev Psychobiol 2021;63:973-84.

18 Letourneau NL, Kozyrskyj AL, Cosic N, et al. Maternal sensitivity and social support protect against childhood atopic dermatitis. Allergy Asthma Clin Immunol 2017;13:1-14.

19 Giesbrecht Get al. The buffering effect of social support on HPA axis function during pregnancy. Psychosom Med 2013;75:856-62.

20 Giesbrecht GF, Campbell T, Letourneau N, et al. Sexually dimorphic adaptations in basal maternal stress physiology during pregnancy and implications for fetal development. Psychoneuroendocrinology 2015;56:168-78.

21 Giesbrecht GF, Poole JC, Letourneau N, et al. The buffering effect of social support on hypothalamic-pituitary-adrenal axis function during pregnancy. Psychosom Med 2013;75:856-62.

22 Thomas-Argyriou JCet al. The role of HPA-axis function during pregnancy in the intergenerational transmission of maternal adverse childhood experiences to child behavior problems. Development and Psychopathology 2020:1-17.

23 Giesbrecht GF, Campbell T, Letourneau N, et al. Psychological distress and salivary cortisol covary within persons during pregnancy. Psychoneuroendocrinology 2012;37:270-9.

24 Thomas JC, Letourneau N, Bryce Cl, et al. Biological embedding of perinatal social relationships in infant stress reactivity. Dev Psychobiol 2017:59:425-35.

25 Rash JA, Campbell TS, Letourneau N, et al. Maternal cortisol during pregnancy is related to infant cardiac vagal control. Psychoneuroendocrinology 2015;54:78-89.

26 Thomas JC, Letourneau N, Campbell TS, et al. Developmental origins of infant emotion regulation: mediation by temperamental negativity and moderation by maternal sensitivity. Dev Psychol 2017:53:611-28.

27 Giesbrecht GF, Campbell T, Letourneau N, et al. Advancing gestation does not attenuate biobehavioural coherence between psychological distress and cortisol. Biol Psychol 2013;93:45-51.

28 Leung BMY, Kaplan BJ, Field CJ, et al. Prenatal micronutrient supplementation and postpartum depressive symptoms in a pregnancy cohort. BMC Pregnancy Childbirth 2013;13:2.

29 Letourneau N, Dewey D, Kaplan BJ, et al. Intergenerational transmission of adverse childhood experiences via maternal depression and anxiety and moderation by child sex. J Dev Orig Health Dis 2019;10:88-99.

30 Leung BMY, Letourneau NL, Giesbrecht GF, et al. Predictors of postpartum depression in partnered mothers and fathers from a longitudinal cohort. Community Ment Health J 2017;53:420-31.

31 Field T, Diego M, Hernandez-Reif M, et al. Comorbid depression and anxiety effects on pregnancy and neonatal outcome. Infant Behav Dev 2010;33:23-9.

32 Letourneau NL, Tramonte L, Willms JD. Maternal depression, family functioning and children's longitudinal development. J Pediatr Nurs 2013;28:223-34.

33 Yim IS, Tanner Stapleton LR, Guardino CM, et al. Biological and psychosocial predictors of postpartum depression: systematic review and call for integration. Annu Rev Clin Psychol 2015;11:99-137.

34 Guintivano J, Manuck T, Meltzer-Brody S. Predictors of postpartum depression: a comprehensive review of the last decade of evidence. Clin Obstet Gynecol 2018;61:591-603.

35 Bergink V, Kooistra L, Lambregtse-van den Berg MP, et al. Validation of the Edinburgh depression scale during pregnancy. J Psychosom Res 2011;70:385-9.

36 Paulson JF, Bazemore SD. Prenatal and postpartum depression in fathers and its association with maternal depression: a metaanalysis. JAMA 2010;303:1961-9.

37 Gavin NI, Gaynes BN, Lohr KN, et al. Perinatal depression: a systematic review of prevalence and incidence. Obstet Gynecol 2005;106:1071-83.

38 Carlberg M, Edhborg M, Lindberg L. Paternal perinatal depression assessed by the Edinburgh postnatal depression scale and the Gotland male depression scale: prevalence and possible risk factors. Am J Mens Health 2018:12:720-9.

39 Pasco JA, Jacka FN, Williams LJ, et al. Dietary selenium and major depression: a nested case-control study. Complement Ther Med 2012;20:119-23.

40 Markhus MW, Skotheim S, Graff IE, et al. Low omega-3 index in pregnancy is a possible biological risk factor for postpartum depression. PLoS One 2013;8:e67617.

41 Felitti VJ, Anda RF, Nordenberg D, et al. Relationship of childhood abuse and household dysfunction to many of the leading causes of death in adults: The adverse childhood experiences (ACE) study. Am J Prev Med 1998;14:245-58.

42 Danese A, Moffitt TE, Harrington H, et al. Adverse childhood experiences and adult risk factors for age-related disease: depression, inflammation, and clustering of metabolic risk markers. Arch Pediatr Adolesc Med 2009;163:1135-43.

43 Hughes K, Bellis MA, Hardcastle KA, et al. The effect of multiple adverse childhood experiences on health: a systematic review and meta-analysis. Lancet Public Health 2017;2:e356-66.

44 Begum F, Colman I, McCargar LJ, et al. Gestational weight gain and early postpartum weight retention in a prospective cohort of Alberta women. J Obstet Gynaecol Can 2012;34:637-47.

45 Jarman M, Yuan Y, Pakseresht M, et al. Patterns and trajectories of gestational weight gain: a prospective cohort study. CMAJ Open 2016:4:E338-45.

46 Subhan FB, Shulman L, Yuan Y, et al. Association of pre-pregnancy $\mathrm{BMI}$ and gestational weight gain with fat mass distribution and accretion during pregnancy and early postpartum: a prospective study of Albertan women. BMJ Open 2019;9:e026908.

47 Jarman M, Mathe N, Ramazani F, et al. Dietary patterns prior to pregnancy and associations with pregnancy complications. Nutrients 2018;10. doi:10.3390/nu10070914. [Epub ahead of print: $17 \mathrm{Ju}$ 2018].

48 Aghajafari F, Field CJ, Rabi D, et al. Plasma 3-Epi-25Hydroxycholecalciferol can alter the assessment of vitamin D status using the current reference ranges for pregnant women and their newborns. J Nutr 2016;146:70-5.

49 Aghajafari F, Field CJ, Kaplan BJ, et al. The current recommended vitamin $D$ intake guideline for diet and supplements during pregnancy is not adequate to achieve vitamin $\mathrm{D}$ sufficiency for most pregnant women. PLOS One 2016;11:e0157262.

50 Aghajafari F, Field CJ, Kaplan BJ, et al. The high prevalence of vitamin D insufficiency in cord blood in Calgary, Alberta (APrON-D study). J Obstet Gynaecol Can 2017;39:347-53.

51 Lewis ED, Subhan FB, Bell RC, et al. Estimation of choline intake from $24 \mathrm{H}$ dietary intake recalls and contribution of egg and milk consumption to intake among pregnant and lactating women in Alberta. Br J Nutr 2014;112:112-21.

52 Goverment of Canada. Prenatal nutrition guidelines for health professionals: gestational weight gain, 2010. Available: https://www. canada.ca/en/health-canada/services/canada-food-guide/resources/ prenatal-nutrition/eating-well-being-active-towards-healthy-weightgain-pregnancy-2010.html [Accessed 21 Jan 2020].

53 APrON and ENRICH Study Teams. Adherence to Canada's food guide recommendations during pregnancy: nutritional epidemiology and public health. Curr Dev Nutr 2017;1:e000356.

54 Aghajafari $\mathrm{F}$, et al, Association between maternal serum 25-hydroxyvitamin D level and pregnancy and neonatal outcomes. Systematic review and meta-analysis of observational studies. $\mathrm{Bm}$ 2013;346:f1169.

55 Wallace TC, Blusztajn JK, Caudill MA, et al. Choline: the neurocognitive essential nutrient of interest to obstetricians and Gynecologists. J Diet Suppl 2020;17:1-20.

56 Bresson JL, Burlingame B, Dean T, et al. Dietary reference values for choline EFSA panel on dietetic products. EFSA Journal 2016;14:4484.

57 Subhan FB, Colman I, McCargar L, et al. Higher pre-pregnancy $\mathrm{BMI}$ and excessive gestational weight gain are risk factors for rapid weight gain in infants. Matern Child Health J 2017;21:1396-407.

58 Aghajafari F, Field C, Weinberg A, et al. Both mother and infant require a vitamin $D$ supplement to ensure that infants' vitamin $D$ status meets current guidelines. Nutrients 2018;10:429.

59 Letourneau N, Leung B, Ntanda $\mathrm{H}$, et al. Maternal and paternal perinatal depressive symptoms associate with 2- and 3-year-old children's behaviour: findings from the APrON longitudinal study. BMC Pediatr 2019;19:435.

60 Sommer A, Twig G. The impact of childhood and adolescent obesity on cardiovascular risk in adulthood: a systematic review. Curr Diab Rep 2018;18:1-6.

61 Sacco MR, de Castro NP, Euclydes VLV, et al. Birth weight, rapid weight gain in infancy and markers of overweight and obesity in childhood. Eur J Clin Nutr 2013;67:1147-53.

62 Ghoorah K, Campbell P, Kent A, et al. Obesity and cardiovascular outcomes: a review. Eur Heart $J$ Acute Cardiovasc Care 2016:5:77-85.

63 Mailhot G, White JH. Vitamin D and immunity in infants and children. Nutrients 2020;12:1233

64 Arman S. What are the effects of vitamin D supplementation for term breastfed infants to prevent vitamin $D$ deficiency and improve bone health? - A Cochrane Review summary with commentary. $J$ Musculoskelet Neuronal Interact 2021;21:193. 
65 Aronen ET, Soininen M. Childhood depressive symptoms predict psychiatric problems in young adults. Can J Psychiatry 2000;45:465-70.

66 Benjet C, Albor YC, Bocanegra ES, et al. Incidence and recurrence of depression from adolescence to early adulthood: a longitudinal follow-up of the Mexican adolescent mental health survey. $J$ Affect Disord 2020;263:540-6.

67 Copeland WE, Shanahan L, Costello EJ, et al. Childhood and adolescent psychiatric disorders as predictors of young adult disorders. Arch Gen Psychiatry 2009;66:764-72.

68 Hege A, Bouldin E, Roy M, et al. Adverse childhood experiences among adults in North Carolina, USA: influences on risk factors for poor health across the lifespan and intergenerational implications. Int $J$ Environ Res Public Health 2020;17:8548.

69 Sheffler JL, Stanley I, Sachs-Ericsson N. ACEs and mental health outcomes, in adverse childhood experiences. Elsevier, 2020: 47-69.

70 Vig KD, Paluszek MM, Asmundson GJ. ACEs and physical health outcomes, in adverse childhood experiences. Elsevier, 2020: 71-90.
71 McDonald SW, Madigan S, Racine N, et al. Maternal adverse childhood experiences, mental health, and child behaviour at age 3: the all our families community cohort study. Prev Med 2019;118:286-94.

72 Racine N, Plamondon A, Madigan S, et al. Maternal adverse childhood experiences and infant development. Pediatrics 2018;141. doi:10.1542/peds.2017-2495. [Epub ahead of print: 20 03 2018].

73 Letourneau N, Duffett-Leger L, Salmani M. The role of paternal support in the behavioural development of children exposed to postpartum depression. Can J Nurs Res 2009;41:86-106.

74 Rao W-W, Zhu X-M, Zong Q-Q, et al. Prevalence of prenatal and postpartum depression in fathers: a comprehensive meta-analysis of observational surveys. J Affect Disord 2020;263:491-9.

75 Daniels JL, Savitz DA, Bradley C, et al. Attitudes toward participation in a pregnancy and child cohort study. Paediatr Perinat Epidemiol 2006;20:260-6. 